\title{
Toward the Identification of Potential $\alpha$-Ketoamide Covalent Inhibitors for SARS-CoV-2 Main Protease: Fragment-Based Drug Design and MM-PBSA Calculations
}

\author{
Mahmoud A. El Hassab ${ }^{1}$, Mohamed Fares ${ }^{2,3}$, Mohammed K. Abdel-Hamid Amin 4 ${ }^{\mathbb{D}}$, Sara T. Al-Rashood 5,* \\ Amal Alharbi ${ }^{5}$, Razan O. Eskandrani ${ }^{5}$, Hamad M. Alkahtani ${ }^{5}$ (D) and Wagdy M. Eldehna ${ }^{6}(\mathbb{D}$ \\ 1 Department of Pharmaceutical Chemistry, School of Pharmacy, Badr University in Cairo (BUC), \\ Cairo 11829, Egypt; mahmoud65582@pharm.tanta.edu.eg \\ 2 Department of Pharmaceutical Chemistry, Faculty of Pharmacy, Egyptian Russian University, \\ Badr 11829, Egypt; mm342@uowmail.edu.au \\ 3 School of Chemistry, The University of Sydney, Sydney, NSW 2006, Australia \\ 4 School of Medicine, The University of Notre Dame Australia, Fremantle, WA 6160, Australia; \\ mkah425@uowmail.edu.au \\ 5 Department of Pharmaceutical Chemistry, College of Pharmacy, King Saud University, \\ Riyadh 11451, Saudi Arabia; amal.harbi@gmail.com (A.A.); Razan.a.esk@gmail.com (R.O.E.); \\ ahamad@ksu.edu.sa (H.M.A.) \\ check for \\ updates \\ Citation: Hassab, M.A.E.; Fares, M.; \\ Amin, M.K.A.-H.; Al-Rashood, S.T.; \\ 6 Department of Pharmaceutical Chemistry, Faculty of Pharmacy, Kafrelsheikh University, \\ Kafrelsheikh 33516, Egypt; wagdy2000@gmail.com \\ * Correspondence: salrashood@ksu.edu.sa
} Alharbi, A.; Eskandrani, R.O.; Alkahtani, H.M.; Eldehna, W.M. Toward the Identification of Potential $\alpha$-Ketoamide Covalent Inhibitors for SARS-CoV-2 Main Protease: Fragment-Based Drug Design and MM-PBSA Calculations. Processes 2021, 9, 1004. https://doi.org/ $10.3390 /$ pr9061004

Academic Editors: Yunfei Du, Teodorico De Castro Ramalho, Zhong-Ru Xie and Kuan Y. Chang

Received: 29 April 2021

Accepted: 1 June 2021

Published: 5 June 2021

Publisher's Note: MDPI stays neutral with regard to jurisdictional claims in published maps and institutional affiliations.

Copyright: (c) 2021 by the authors Licensee MDPI, Basel, Switzerland. This article is an open access article distributed under the terms and conditions of the Creative Commons Attribution (CC BY) license (https:// creativecommons.org/licenses/by/ $4.0 /)$.

Abstract: Since December 2019, the world has been facing the outbreak of the SARS-CoV-2 pandemic that has infected more than 149 million and killed 3.1 million people by 27 April 2021, according to WHO statistics. Safety measures and precautions taken by many countries seem insufficient, especially with no specific approved drugs against the virus. This has created an urgent need to fast track the development of new medication against the virus in order to alleviate the problem and meet public expectations. The SARS-CoV-2 3CL main protease (Mpro) is one of the most attractive targets in the virus life cycle, which is responsible for the processing of the viral polyprotein and is a key for the ribosomal translation of the SARS-CoV-2 genome. In this work, we targeted this enzyme through a structure-based drug design (SBDD) protocol, which aimed at the design of a new potential inhibitor for Mpro. The protocol involves three major steps: fragment-based drug design (FBDD), covalent docking and molecular dynamics (MD) simulation with the calculation of the designed molecule binding free energy at a high level of theory. The FBDD step identified five molecular fragments, which were linked via a suitable carbon linker, to construct our designed compound RMH148. The mode of binding and initial interactions between RMH148 and the enzyme active site was established in the second step of our protocol via covalent docking. The final step involved the use of MD simulations to test for the stability of the docked RMH148 into the Mpro active site and included precise calculations for potential interactions with active site residues and binding free energies. The results introduced RMH148 as a potential inhibitor for the SARS-CoV-2 Mpro enzyme, which was able to achieve various interactions with the enzyme and forms a highly stable complex at the active site even better than the co-crystalized reference.

Keywords: SARS-CoV-2 main protease inhibitor; COVID-19 treatment; structure-based drug design; molecular docking; molecular dynamics

\section{Introduction}

Emerging and neglected viral diseases are of immediate and critical societal importance as exemplified by the current COVID-19 pandemic. The virus has been reported worldwide with more than 149 million confirmed cases and more than 3.1 million deaths 
as of February 2021. So far, there is no specific antiviral drug for COVID-19 infection, and drug development and research have been limited to certain drug repurposing studies [1]. Despite the restrictions and safety measures that have been applied worldwide, the spread of the SARS-CoV-2 virus is still very hard to control, which highlights the urgent need to develop potent drugs against the virus. SARS-CoV-2 belongs to the coronavirus family, which is a single, positive-stranded RNA that is implicated in many human diseases, especially respiratory and neurological diseases [2]. The coronaviruses were the primary cause for two major outbreaks: in 2002, the severe acute respiratory syndrome coronavirus (SARS); and in 2012, the Middle East respiratory syndrome (MERS) [3,4].

It is very difficult and challenging to develop a specific treatment for COVID-19 as a result of many obstacles that slow or even cease the cycle of drug discovery. A major one of those obstacles is the lack of proper biological investigations and experimental facilities for COVID-19, probably due to the risk of infection from the virus and the restrictions taken by many countries.

Extensive studies investigating the SARS-CoV-2 life cycle revealed many potential targets for drug discovery against the COVID-19 infection, including the main protease (Mpro), angiotensin-converting enzyme II (ACE2) entry receptor, and the RNA-dependent RNA polymerase (RdRp) (Figure 1) [5-7]. Inhibitors for COVID-19 Mpro are thought to be safer and more effective, as they are very distinct from human proteases, unlike ACE2 and RdRp inhibitors, which may result in serious side effects [8,9].

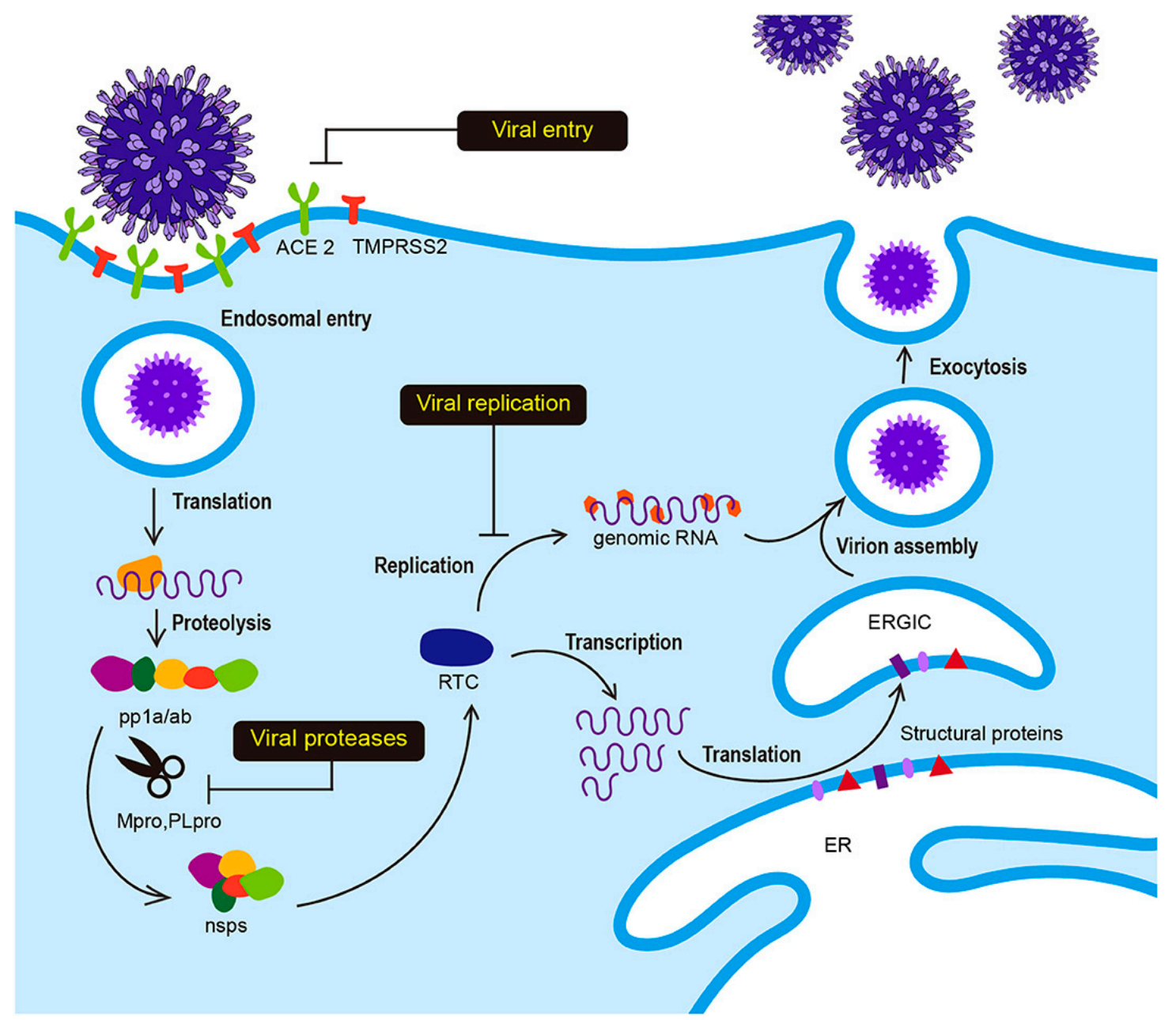

Figure 1. Life cycle of SARS-CoV-2 nonstructural proteins (RNA-dependent-RNA polymerase and hellicase) (nsps), replicase-transcriptase complex (RTC), endoplasmic reticulum (ER), nucleocapsid (N), ER-golgi intermediate compartment (ERGIC), co-terminal polyproteins (pp1a/ab). Angiotensin-converting enzyme (ACE 2). Reproduced with permission from Jeong, G.U.; Song, H.; Yoon, G.Y.; Kim, D.; Kwon, Y.-C., Frontiers in. Microbiology, published by frontiers, 2020 [7]. 
The Mpro has a vital role in SARS-CoV-2 replication by processing the polyprotein resulting from the RNA transcription into functioning units essential for virus replication, maturation and survival [10]. The Mpro cleavages eleven specific sites containing glutamine residues in the viral polyprotein, which are not reported in any human protease [11-13]. Its activity relies on the activation of two catalytic dyads, cysteine and histidine, in the active site. Initially, the thiol of the cysteine residue is activated to thiolate anion through proton abstraction by the histidine residue. This is followed by the nucleophilic attack of the thiolate anion on the peptide bond forming a thioester bond. The thioester is hydrolyzed, and the thiol group is restored after proton abstraction from the / histidine imidazole. Finally, the process is repeated until the cleavage of the entire polyprotein $[14,15]$ (Figure S1).

Many viral protease inhibitors were designed based on trapping the catalytic triad or dyad of the protease enzymes. Examples for these inhibitors include the FDA-approved HCV protease inhibitors, such assimeprevir and grazoprevir [16] in addition to commercially available HIV protease inhibitors, such as ritonavir and lopinavir [17]. All the mentioned inhibitors contain either acyl sulphonamide or alpha keto-amide as a trapping group for the cysteine or serine residues [18]. This emphasizes the importance of considering these moieties when designing an effective, potent and specific inhibitor for Mpro.

Fragment-based drug design FBDD (fragment based drug design) is an inevitable computer-aided drug design strategy that is especially useful when the structure of the target is available while there is limited knowledge about potential ligands [19]. Through the screening of the fragments database in the active site of the target, the technique enables the discovery of novel drugs, overcoming many obstacles resulted from the lack of sufficient data. In general, the superiority of FBDD on other virtual high-throughput screening (HTS) strategies includes the ability to fully explore the enzyme active site, which is enabled by the small fragments that are involved in FBDD that could be easily inserted in any space in the active site, in contrast to large molecules from other HTS methods [20,21]. This advantage allows for higher hit rates as well as higher binding affinity, as compared to conventional HTS [22,23].

Given our continuous efforts in the development of COVID-19 inhibitors [24], we were keen to employ a modeling approach to the rational design of a new COVID-19 Mpro inhibitor. Herein, we report on our efforts to utilize the reported crystal structure of COVID-19 Mpro (PDB ID: 6y2g) and FBDD and docking and molecular dynamics (MD) techniques to identify a novel chemical entity against the COVID-19 Mpro enzyme.

\section{Results}

\subsection{Fragment-Based Drug Design (FBDD)}

The employment of FBDD is based on three strategies: (a) fragment merging [25] where two fragments oriented to the same regions in the binding site of the target are merged to give a one desired fragment; (b) fragment linking [25] where two or more fragments, interacting with different domains in the binding site of the target, are linked by suitable linkers to yield single compound; and (c) fragment growing [25] where a fragment matched with the role of three is increased in size via attaching with different fragments to optimize and increase the interaction of the proposed target.

Our design for a novel COVID-19 Mpro inhibitor was based on both fragment growing and linking strategies, using the de novo receptor protocol of the DS software. The alpha keto amide moiety was used as the principle fragment, and the active site was determined by a cavity surrounding the binding of the co-crystalized ligand. Utilizing this technique, we were able to subdivide the active site cavity and allow the fragments to be screened through the entire binding domain. The Ludi database of the DS software that contains 1053 diverse fragments with a molecular weight less than $300 \mathrm{KD}$ was used as the source of fragments for the screening, and the "Energy estimate 3" algorithm was used as a scoring function. Fragments that showed good affinity to the receptor as evidenced by a negative change in the free energy upon binding to the receptor were allowed to pass 
the scoring filter, and the process resulted in the identification of 618 fragments. Further fragment filtration was performed by docking each fragment into the receptor cavity, using the MCSS (multiple copy simultaneous search ) algorithm [26]. Visual inspection of the bound fragments revealed potentially strong binding with the enzyme and the involvement of multiple interactions at the active site (Figure 2a). These fragments were then linked together and to the alpha keto-amide via a carbon linker to construct the designed compound RMH148 (Figure 2b).

a) (ำ

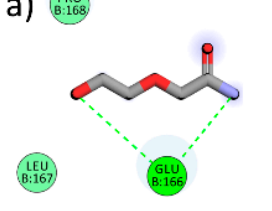

F1

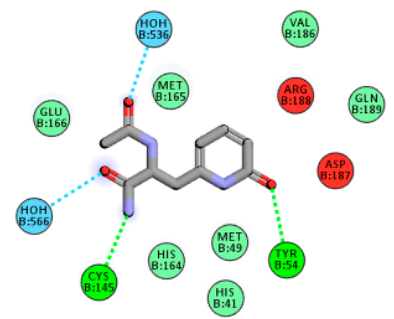

F2

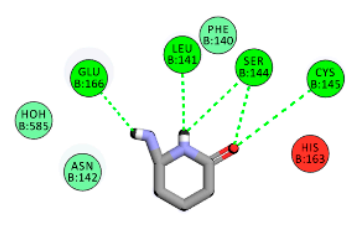

(19:010

F3

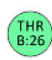

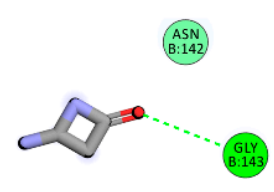

F4

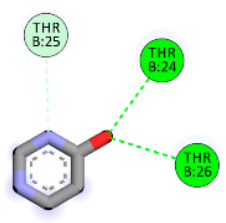

F5

b)

c)

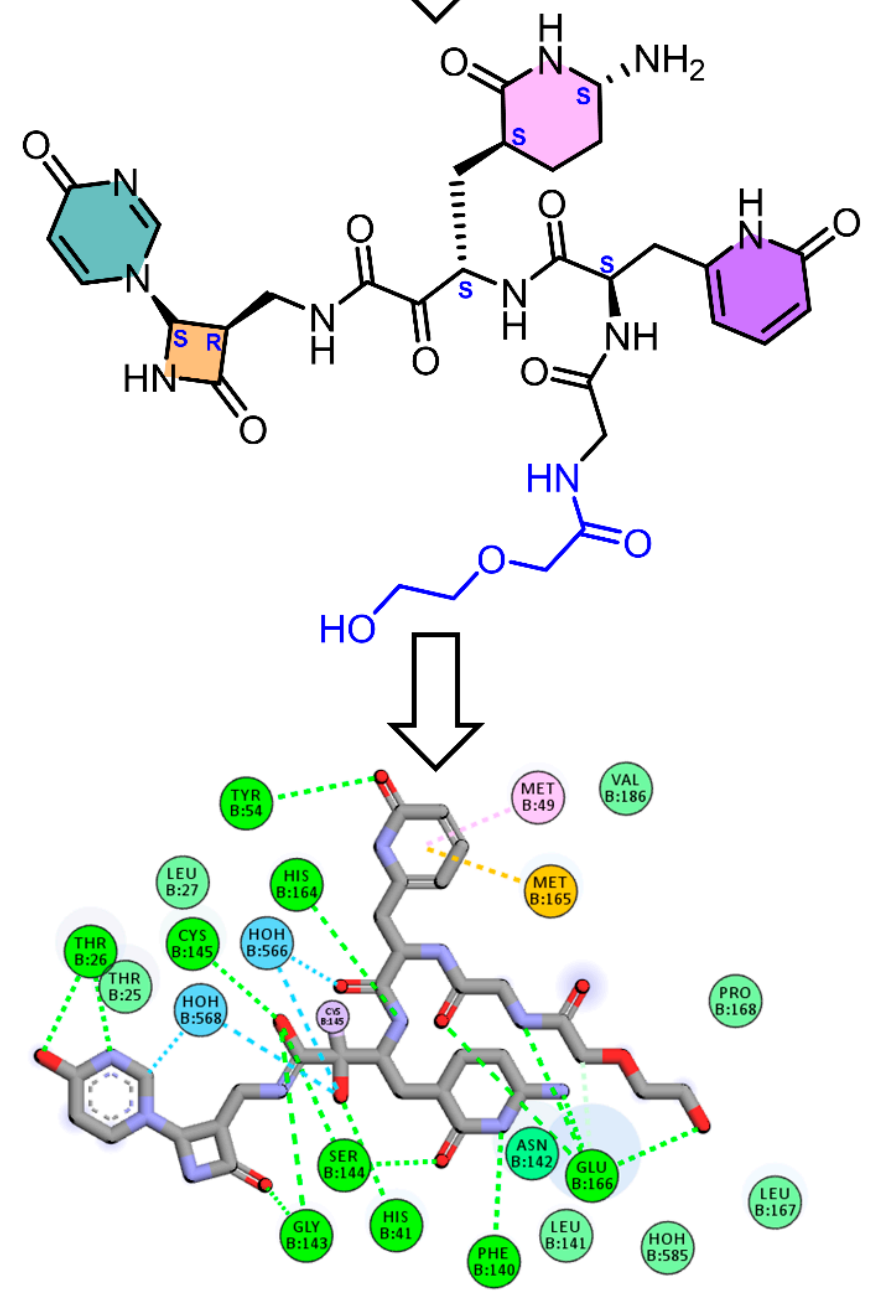

Figure 2. (a) The five generated fragments from FBDD, (b) the designed compound RMH148 after fragment linking, (c) the 2d binding diagram between RMH148 and Mpro. 


\subsection{Covalent Docking}

Our next step was to confirm the binding mode of RMH148 into the COVID-19 Mpro active site by applying a covalent docking simulation, using DOCKTITE wizard of the MOE (Version: 2019.0102, chemical computing group, Montreal, Quebec, Canada 2019) software. This protocol was specifically applied, keeping in mind the challenges for protease inhibitors docking, which usually involves bond breaking and new bond formation. DOCKTITE wizard uses a highly versatile workflow for covalent docking and enables the prediction of covalent interactions by the determination of the electrophilic site of the ligand and the nucleophilic site of the receptor with the ability to determine the reaction type and the formed product. During the docking simulation, RMH148 was placed into the active site, using the GBVI/WSA dG (generalized born volume integral) scoring function. The carbon atom of the alpha carbonyl of the ligand and the thiolate of cysteine145 in COVID-19 Mpro were selected as the electrophilic site of the ligand and the nucleophilic site of the receptor, respectively. The validation of the protocol was performed by re-docking the co-crystalized ligand (O6K) to the COVID-19 Mpro, using the same parameters, which resulted in a calculated RMSD (root mean square deviation) between the docked and co-crystalized poses of O6K of $1.2 \AA$ (Figure 3).

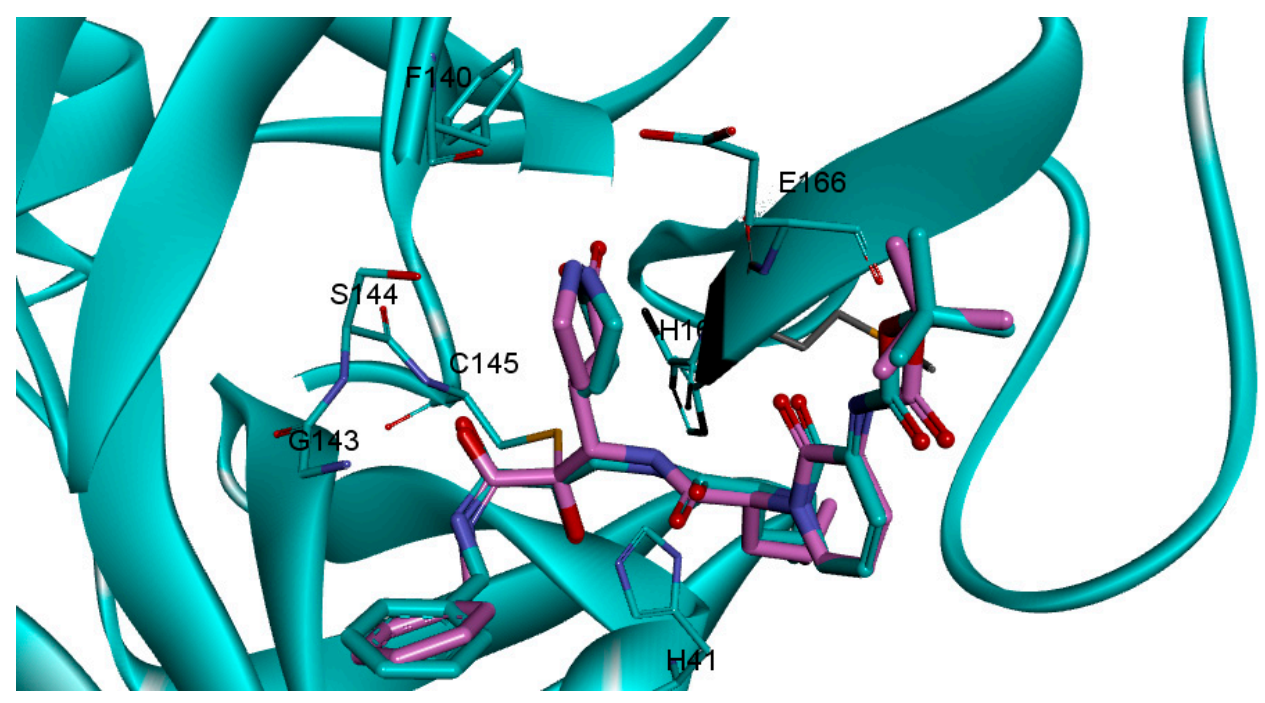

Figure 3. The re-docked pose (cyan) superimposed on the co-crystalized (pink).

The docking results showed binding scores of -7.1 and -10.30 for O6K and RMH148, respectively, which indicates the potential for stronger binding and more interactions later with the COVID-19 Mpro. A closer look at the docked RMH148, as depicted in Figure 4, shows its engagement in a number of diverse interaction types within the binding site. For instance, RMH148 was able to make hydrogen-bond interactions with residues THR26, HIS41, TYR54, PHE140, GLY143, SER144, CYS145, HIS164 and GLU166, in addition to a covalent bond with residue CYS145. Moreover, RMH148 achieved several hydrophobic interactions with residues MET49 and MET165. The detailed interactions of RMH148 within the COVID-19 Mpro active site are summarized in the supporting information in Table S1. 


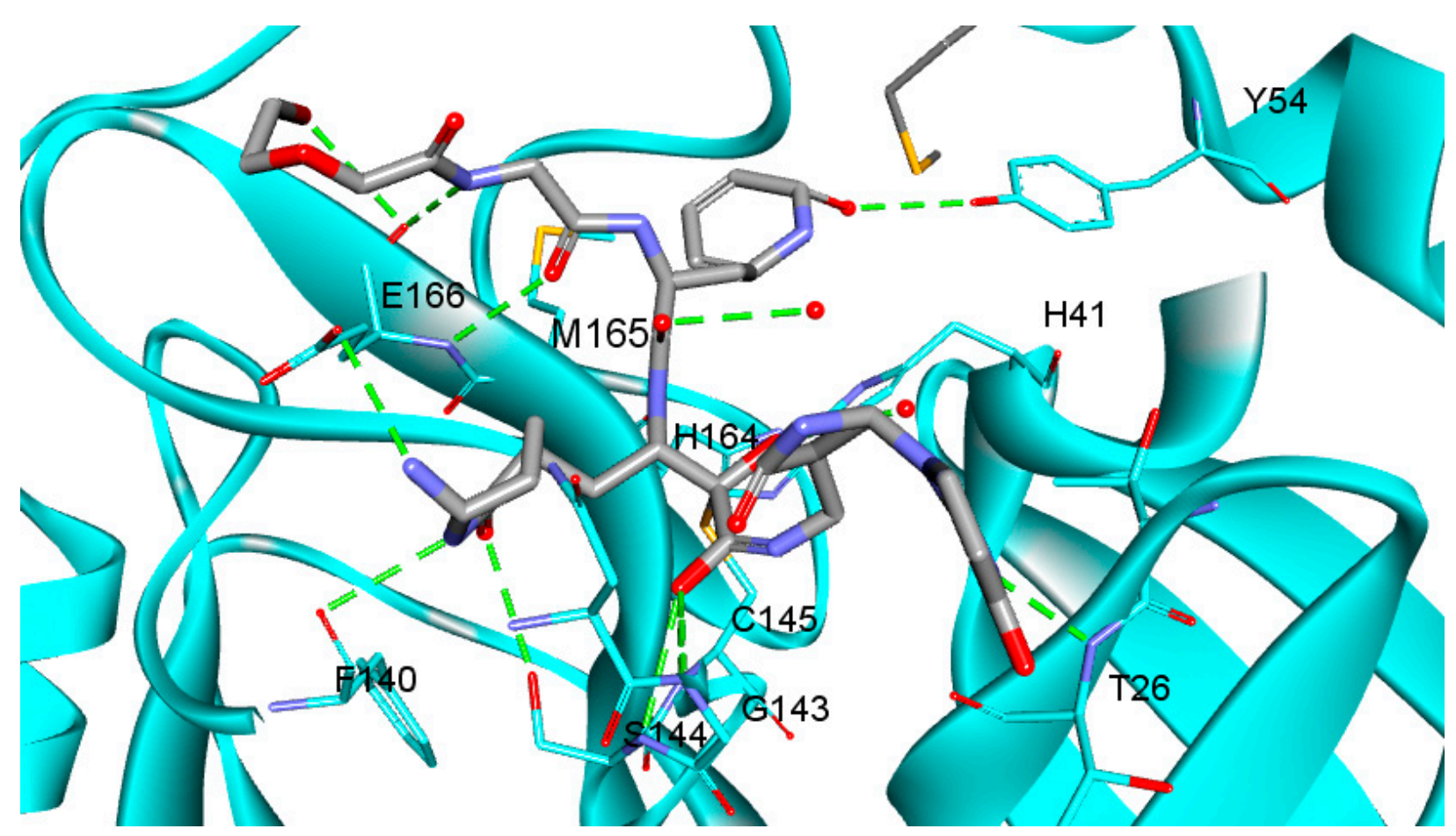

(A)

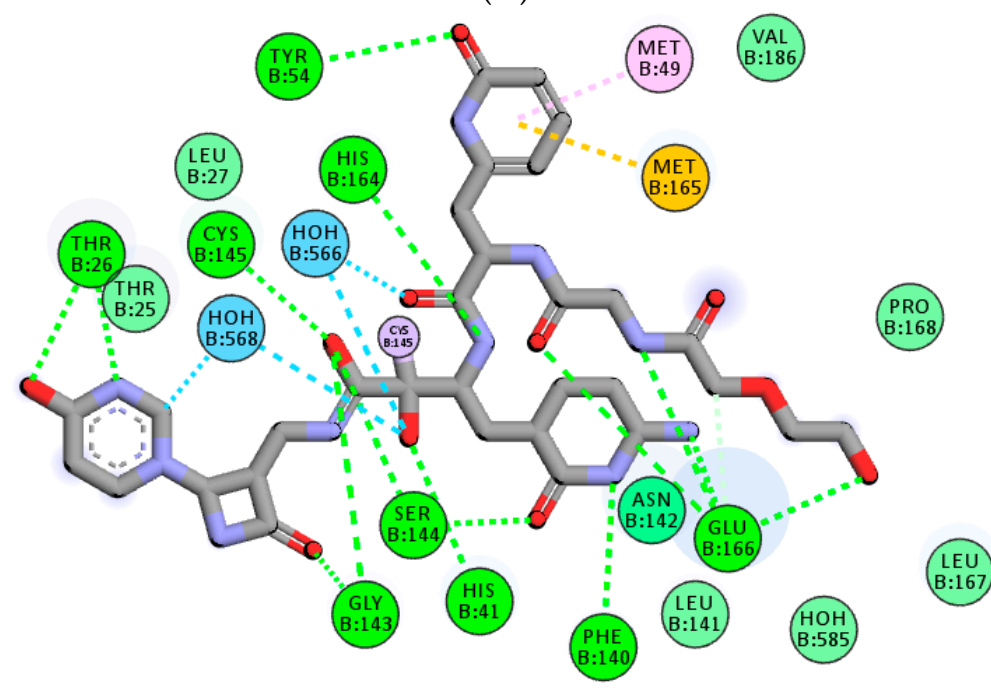

(B)

Figure 4. The 3D (A) and 2D (B) interaction diagram between RMH148 and the Mpro enzyme; the ligand is shown in stick presentation. Relevant residues at the active site are shown in line presentations. Bond lengths between the ligand and interacting residues are shown in the 3D diagram.

\subsection{In Silico ADME and Toxicity Calculations}

Drug candidates should have both desired pharmacokinetic and pharmacodynamic properties in addition to a high safety margin. Therefore, the physicochemical properties and potential toxicity of RMH148 were predicted by the online servers Swiss ADME and Preadmet, respectively. Swiss ADME predicted RMH148 to have no inhibitory activity on any of the cytochrome enzymes family. This important criterion enables the safe use of RMH148 concurrently with all the members of the COVID-19 protocol without the concerns of any drug-drug interactions. RMH148 was predicted to have no CNS or placental penetration, due its high polarity, making it an excellent candidate for pregnant patients and patients with neurological disorders. The Preadmet server predicted RMH148 
to have no mutagenicity or carcinogenicity. Unfortunately, RMH148 suffers a low GIT absorption due to its large MWT (743) and high polarity. However, its higher water solubility enables its administration via the intravenous route, making it suitable for emergency cases. Additionally, it is worthy to mention that RMH148 has no PAINS (pan assay interface structure) alerts. Finally, RMH148 and the reference have a synthetic score of 7.6 and 6.5 , making their synthesis possible but very challenging.

\subsection{Molecular Dynamics (MD) Simulations}

\subsubsection{RMSD and RMSF Analysis}

The function of viral proteases requires a wide range of structural flexibility, especially for amine acid residues at the active site. These residues are responsible for the nucleophilic attack on the sessile peptide bond of the enzyme substrate [27]. Considering the power of MD simulations to test the stability of ligand-enzyme complexes as well as calculating binding free energies [28-30], we employed this technique to check the flexibility of the ligand-free COVID-19 Mpro and to probe the stability of the RMH148-Mpro complex structure obtained from the docking calculations. The two structures were subjected to a standard MD protocol with a production phase of $150 \mathrm{~ns}$.

The RMSD values for the enzyme $\mathrm{C}_{\alpha}$ atoms as well as both RMH148 and O6K heavy atoms for the three simulation experiments were calculated by aligning the MD production phase trajectories with their initial structures. In addition, the RMSF values for the three simulation experiments were calculated. Examination of the data presented in the RMSD and RMSF plots (Figure 5) showed that the unbound enzyme had RMSD and RMSF of 4.12 and $4.03 \AA$, respectively, indicating its high dynamic properties. In addition, the RMSD for CYS145 residue was $4.74 \AA$, which highlights its potential role in the nucleophilic attack on the enzyme substrate (Figure 5A).

In comparison, the RMSD results for the RMH148-Mpro complex showed values of $1.15 \mathrm{~A}^{\circ}$ and $1.34 \AA$ for all the residues of the enzyme and CYS145, respectively, while the O6K-Mpro complex had RMSD values of 1.73 and $1.65 \AA$, respectively (Figure 5B). Moreover, the RMSF values for the RMH148-Mpro complex and O6K-Mpro complex were 1.53 and $1.88 \AA$, respectively (Figure 5C). The lower values of RMSD and RMSF compared to those for the ligand-free enzyme reveals the ability of RMH148 to maintain strong binding with the active site and key residue CYS145, which leads to restricting the flexibility and potentially the activity of the enzyme. Moreover, the MD results support the initial docking results and indicate the potential greater ability of RMH148 to inhibit the Mpro enzyme than the crystal reference O6K.

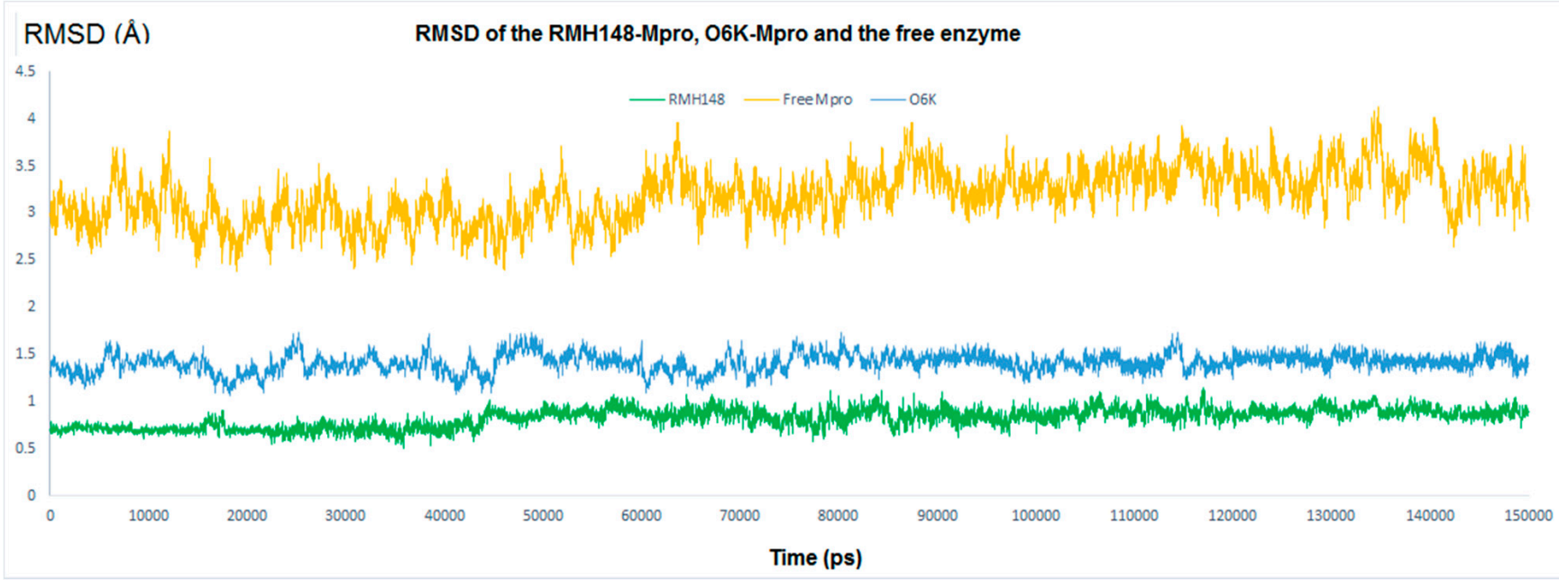

(A)

Figure 5. Cont. 


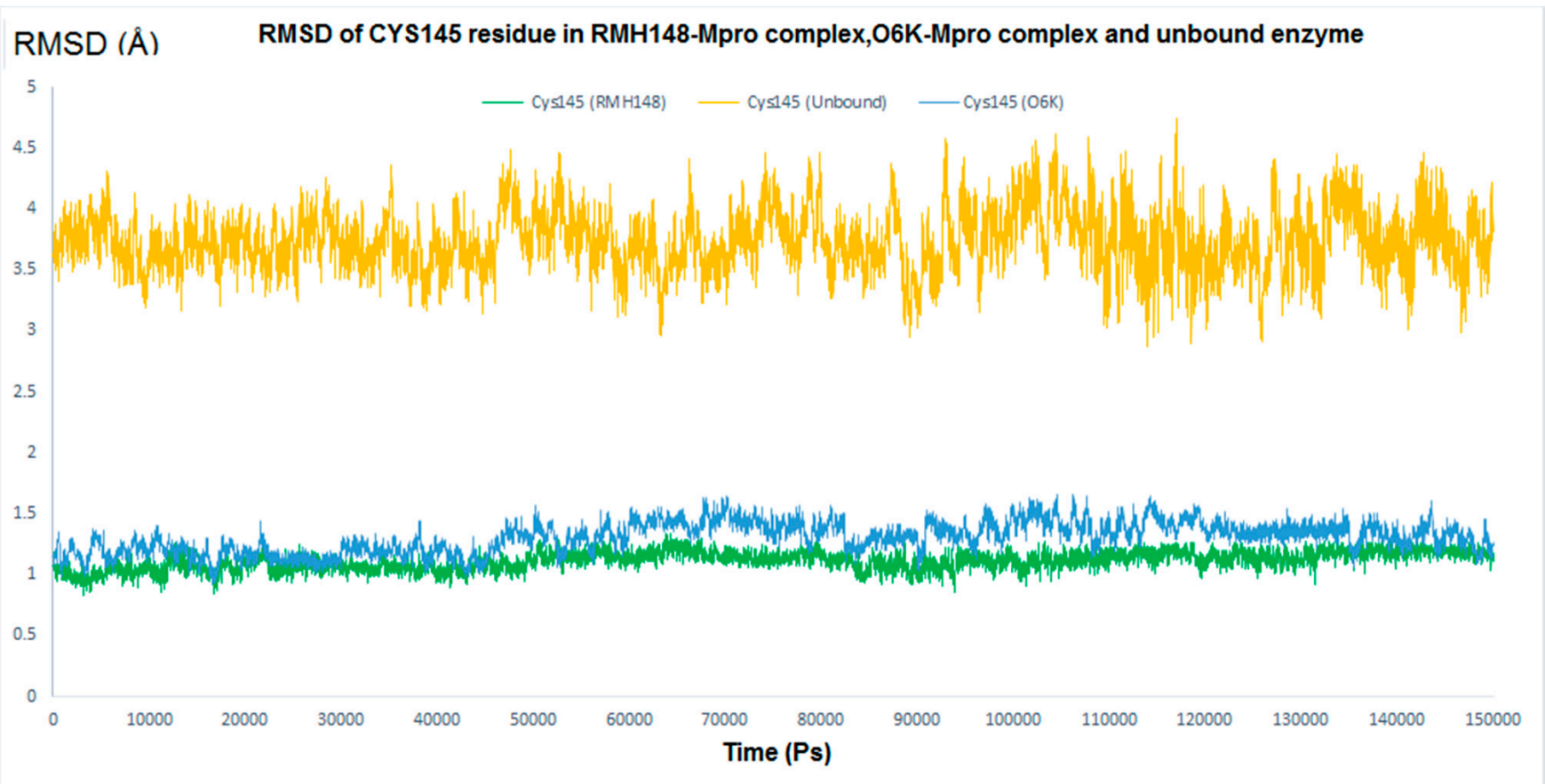

(B)

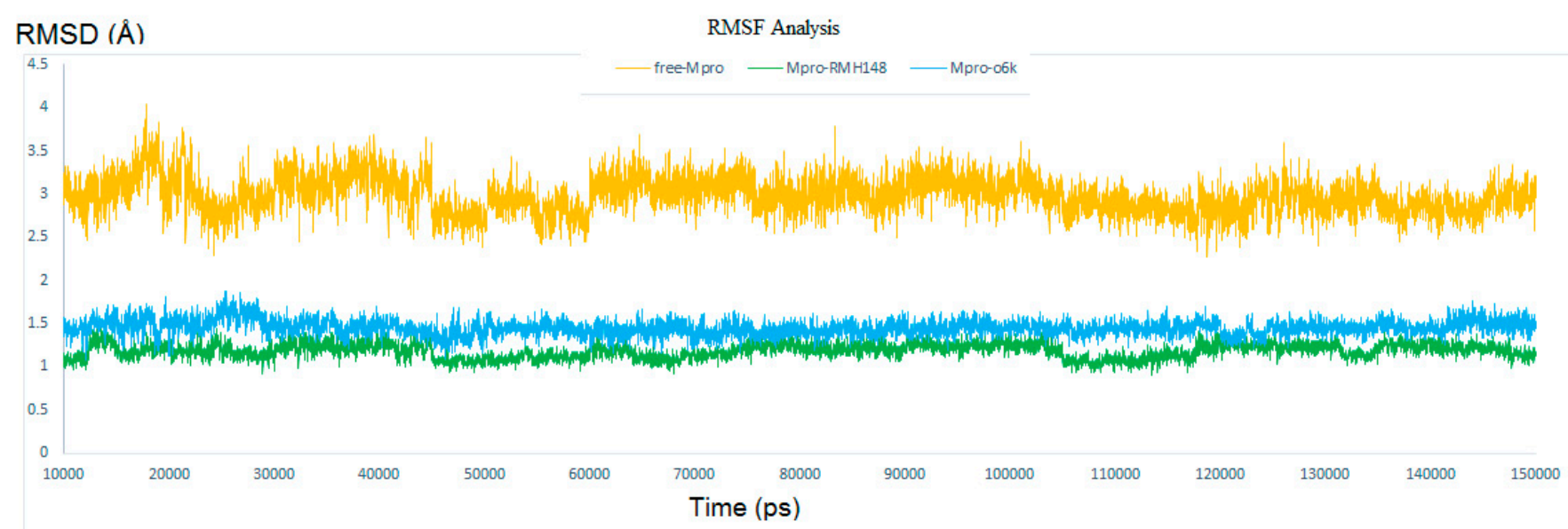

(C)

Figure 5. The RMSD analysis. (A) RMSD for the entire system. Orange color represents Mpro without a ligand, green color represents RMH148-Mpro complex and blue color represents O6K-Mpro complex. (B) RMSD for CYS145, orange color for Mpro without a ligand, green color for RMH148-Mpro complex and blue color for O6K-Mpro complex. (C) RMSF Analysis.

\subsubsection{Evaluating the Stability of RMH148-Mpro}

The binding stability between RMH148 and Mpro was further evaluated by inspecting the MD trajectories for the RMH148-Mpro complex, which reveals multiple stable hydrogen bond interactions throughout the production phase. The bond distances between RMH148 and various COVID-19 Mpro active site residues maintained a value of less than $3.5 \AA$, which indicates the stability of the interaction throughout the simulation (Table 1). 
Table 1. The average distances of all hydrogen bonds formed between RMH148 and COVID-19 Mpro through the entire $150 \mathrm{~ns}$ MD simulation.

\begin{tabular}{cc}
\hline Hydrogen Bond Name & Average Distance $\mathbf{A}^{\mathbf{0}} \mathbf{\text { D }} \pm \mathbf{S D}$ \\
\hline Hydrogen bond with Tyrosine54 & $3.17 \pm 0.06$ \\
Hydrogen bond with Glutamic166 & $3.05 \pm 0.12$ \\
Hydrogen bond with Glutamic166 & $3.05 \pm 0.08$ \\
Hydrogen bond with Glutamic166 & $3.21 \pm 0.1$ \\
Hydrogen bond with Glutamic166 & $3.31 \pm 0.12$ \\
Hydrogen bond with Phenylalanine 140 & $2.98 \pm 0.07$ \\
Hydrogen bond with Serine 144 & $3.17 \pm 0.11$ \\
Hydrogen bond with Serine 144 & $3.18 \pm 0.09$ \\
Hydrogen bond with Glycine 143 & $3.25 \pm 0.15$ \\
Hydrogen bond with Glycine 143 & $3.29 \pm 0.13$ \\
Hydrogen bond with Threonine 26 & $2.43 \pm 0.04$ \\
Hydrogen bond with Threonine 26 & $2.58 \pm 0.17$ \\
Hydrogen bond with Histidine 41 & $3.02 \pm 0.14$ \\
Hydrogen bond with Histidine 164 & $3.00 \pm 0.16$ \\
Hydrogen bond with Cysteine 145 & $2.71 \pm 0.07$ \\
\hline
\end{tabular}

Additionally, the behavior of both RMH148 and O6K in the binding site of the enzyme was monitored by calculating the RMSD of their heavy atoms throughout the entire MD simulations. As described in Figure 6, the RMSD values for RMH148 and O6K were 0.44 and $0.86 \AA$, respectively, indicating a stable binding mode for RMH148. Finally, five conformations from the MD simulations were sampled for the free Mpro and RMH148Mpro that revealed the high stability of RMH148-Mpro in comparison to the free enzymes (supporting information, Figure S3). All of the previous findings attribute the large RMSD values between the free Mpro and RMH148-Mpro to the ability of RMH148 to form many hydrogen bond interactions and covalent bonds with the CYS145 residue, in addition to maintaining those interactions via a stable binding mode.

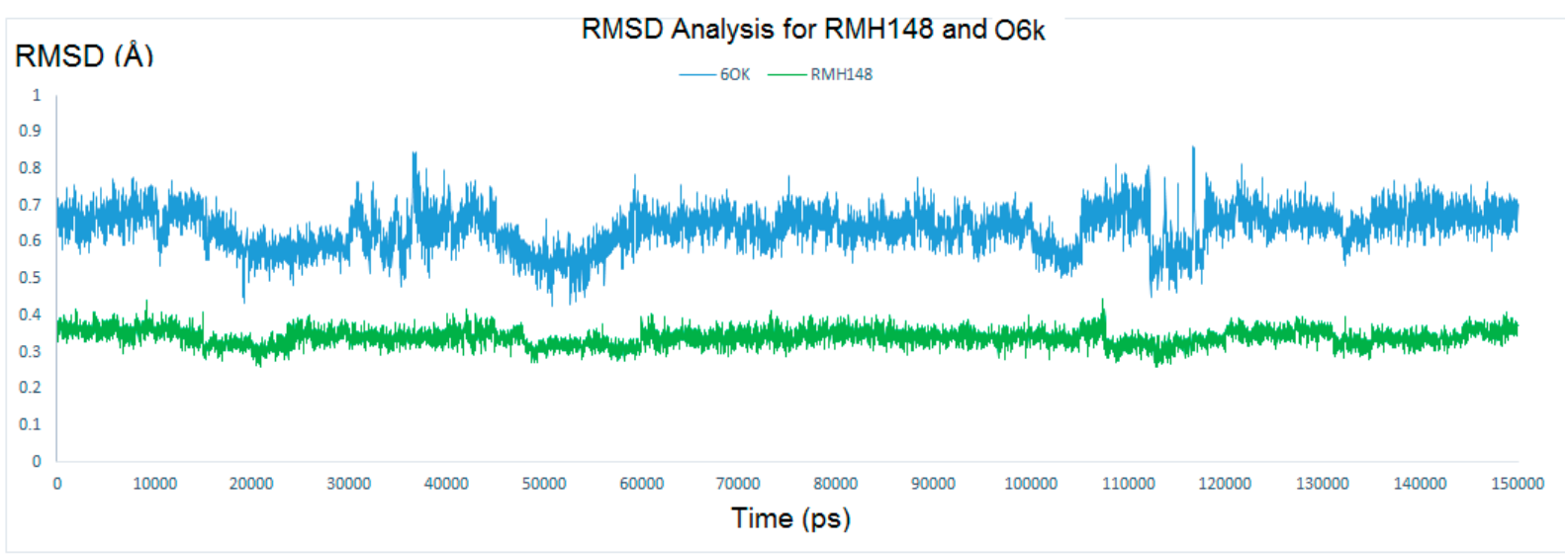

Figure 6. The RMSD of RMH148 in green and O6k in blue.

\subsection{MM-PBSA (Molecular Mechanics Poisson-Boltzmann Surface Area) Binding Free Energy Calculations}

The binding energy for RMH148 and O6K with their potential binding site in the COVID-19 Mpro was calculated, using the MM-PBSA binding free energy methodology. This method represents a more reliable way to calculate the binding free energy through many MD conformations, compared to a single conformation-based score calculated from the docking study. As part of these calculations, the free energy of each component was calculated by summing its molecular mechanics potential energy in a vacuum and the free energy of solvation. The calculated free energy of solvation included the polar solvation energy (electrostatic) and nonpolar solvation energy (non-electrostatic, calculated by the 
solvent accessible surface area; SASA model) [31]. The resulted interactions as well as binding-free energy values are summarized in Table 2 . The data revealed more favorable stability for the RMH148-Mpro complex than the O6K-Mpro complex as indicated by the high negative energy values across the electrostatic, Van der Waal and SASA energies. The only exception was the polar solvation energy, which was in the positive range; however, this can be attributed to the lower solubility of the two complexes, compared to the ligandfree enzyme. Finally, the average binding free energy of the RMH148-Mpro complex was $-412 \mathrm{KJ} / \mathrm{mol}$, which suggests strong and stable binding for RMH148, while the O6K-Mpro complex achieved $-388 \mathrm{KJ} / \mathrm{mol}$ as the average binding free energy. We believe that the results from the MD simulations support our design and validate the entire molecular modeling approach; also, they endorse the potential inhibitory effect of RMH148 on the SARS-CoV-2 Mpro enzyme.

Table 2. The binding free energy for the complexes of RMH148-Mpro and O6k-Mpro, using MMPBSA calculations.

\begin{tabular}{cccccc}
\hline Complex & $\boldsymbol{\Delta} \mathbf{E}_{\text {binding }(\mathbf{k j} / \mathbf{m o l})}$ & $\boldsymbol{\Delta} \mathbf{E}_{\text {Electrostatic }(\mathbf{k j} / \mathbf{m o l})}$ & $\Delta \mathbf{E}_{\text {Van der Waals' }}$ (kj/mol) & $\Delta \mathbf{E}_{\mathbf{p o l a r ~ s o l v a t i o n ~}(\mathbf{k j} / \mathbf{m o l})}$ & $\mathbf{S A S A}_{(\mathbf{k J} / \mathbf{m o l})}$ \\
\hline RMH148-Mpro & $-420 \pm 21$ & $-149 \pm 18$ & $-365 \pm 26$ & $131 \pm 18$ & $-37 \pm 2$ \\
O6K-Mpro & $-388 \pm 20$ & $-138 \pm 19$ & $-333 \pm 23$ & $117 \pm 16$ & $-34 \pm 2$ \\
\hline
\end{tabular}

\section{Material and Methods}

\subsection{Fragment-Based Drug Design (FBDD)}

The 3D structure of COVID-19 Mpro co-crystalized with the alpha-ketoamide inhibitor (O6K) was downloaded from the protein data bank (PDB ID: 6y2g). The alpha-ketoamide moiety of the inhibitor was kept, while the rest of the compound was deleted. Discovery Studio 2016 (DS) software was used to construct a cavity surrounding the active site [32]. The cavity size was expanded to explore every potential interacting residue within the active site. A fragment-based drug design (FBDD) approach was employed, using the de novo receptor server and the default Ludi fragment library of the DS software in order to search fragments that bind to the predetermined active site of the Mpro [33]. The binding strength of each bound fragment from the previous step was further evaluated b] docking into the receptor cavity, using the multiple copy simultaneous search (MCSS) algorithm implemented in the same software $[26,34]$. Highest ranked fragments were linked to the alpha-ketoamide group, using suitable carbon linkers to produce RMH148; the initial binding mode of the generated compound was elucidated from the docking stage.

\subsection{Covalent Docking}

The optimized COVID-19 Mpro structure was used to perform the subsequent docking step. All docking simulations were conducted using MOE software [35]. The receptor and the ligand were prepared using the standard structure optimization protocol of the software. The receptor was energy minimized and equilibrated for $10 \mathrm{~ns}$ under the GROMOS96 43a1 force field. The active site was set as where the co-crystalized ligand was bound. The docking was performed using a molecular database of RMH148 and following the DOCKTITE protocol of MOE software (covalent docking algorithm) [36]. The alpha ketone group in RMH148 was selected as the reactive warhead group and the residue Cys145 was selected as the reactive site of the receptor [37]. The RMSD between the re-docked pose of ligand O6K and co-crystalized ligand was calculated and used to confirm the docking validity. The DS visualizer, available from Biovia Inc., was used to visualize and analyze the docking results as well as produce $2 \mathrm{D}$ and $3 \mathrm{D}$ interaction images.

\subsection{In Silico ADME and Toxicity Calculations}

RMH148's physicochemical properties and toxicity profile were calculated by running the online servers Swiss ADME (http:/ / www.swissadme.ch/index.php in 20 May 2021) and Preadmet (https:/ / preadmet.bmdrc.kr/ 20 May 2021), respectively. 


\subsection{Molecular Dynamics (MD)}

All molecular dynamics (MD) simulations were performed using the GROningen MAchine for Chemical Simulations GROMACS 2020.3 software developed by (University of Groningen, Royal Institute of Technology and Uppsala University) [38]. Three MD simulation experiments were conducted to validate the results retrieved from the docking step. One experiment was performed on the ligand-free Mpro, while the other two were conducted on the enzyme in complex with the designed RMH148 and the crystal reference O6K. The ligand topologies were generated by the Automated Topology Builder (ATB) and Repository version 3 [39] under the GROMOS96 force field and were joined with the enzyme topology, using the standard published protocol [40]. The typical workflow of the GROMACS enzyme-ligand simulations was applied with the solvation of the three systems, using the single point charge (SPC) water model to add water molecules to the cubic simulation boxes. A total number of 28,008 water molecules were added to the system containing the free Mpro. Similarly, 27,999 and 28,001 water molecules were added for the systems containing RHM1848-Mpro and O6k-Mpro, respectively, to neutralize the cubic box (sized $85 \times 85 \times 85$ ). Six sodium counter-ions were added to each of the three systems. The energy minimization step for the unbound and the complexed enzyme structures was achieved, using the steepest descent minimization algorithm with a maximum of 50,000 steps and $<10.0 \mathrm{~kJ} /$ molforce under the GROMOS96 43a1 force field [41]. Two consecutive equilibration ensembles were conducted on the energy-minimized structures. Beginning with the NVT ensemble (constant number of particles, volume and temperature) $(310 \mathrm{~K})$ was done for $2 \mathrm{~ns}$ followed by the NPT ensemble (constant number of particles, pressure and temperature) for $8 \mathrm{~ns}$. The particle mesh ewald (PME) method with a $12 \AA$ cut-off and $12 \AA$ Fourier spacing were used to get the long-range electrostatic value (40). The two equilibrated systems entered the production stage without any restraints for $150 \mathrm{~ns}$ with a time step of $2 \mathrm{fs}$, and the structural coordinates were saved every $5 \mathrm{ps}$. Both the temperature $(310 \mathrm{~K})$ and the pressure $(1 \mathrm{~atm})$ were regulated throughout the simulation V-rescale weak coupling method (modified Berendsen thermostat) and Parrinello-Rahman method $[42,43]$. The root means square deviation (RMSD) of the entire system and for the Cys145 residue was calculated from the generated trajectories from the production step. To endorse the proposed binding mode of RMH148, various scripts of GROMACS were used to calculate the distances of the formed hydrogen bonds between the receptor and the ligand.

\subsection{MM-PBSA Calculation}

Binding-free energy calculations were performed, using MM-PBSA, which applies the following equation:

$$
\Delta \mathrm{G}_{(\text {Binding })}=\mathrm{G}_{(\text {Complex })}-\mathrm{G}_{(\text {Receptor })}-\mathrm{G}_{(\text {Ligand })}
$$

where $G_{(\text {Complex })}$ is the total free energy of the protein-ligand complex and $G_{(\text {Receptor })}$ and $\mathrm{G}_{(\text {Ligand) }}$ are the total free energies of the isolated protein and ligand in solvent, respectively. The total free energy of any of the three mentioned entities (complex, receptor and ligand) were calculated for all MD trajectories from its molecular mechanics potential energy plus the energy of the solvation, using the g_mmpbsa package implemented in the GROMACS software [31]. Individual energies along with the values of standard deviations were calculated and then summed together to yield the average total free energy of each component. Finally, to calculate the binding-free energy, the total free energy of the receptor and the ligand were subtracted from the total free energy of the complex. These calculations were done for the two complexes of Mpro-RMH148 and Mpro-O6K.

\section{Future Prospects for RMH148}

In this study, we aimed to design a potential inhibitor for the pandemic COVID19 infection and to establish guidance for the development of an effective inhibitor for 
COVID-19 Mpro. RMH148 demonstrated great potentiality and binding affinity for SARSCoV-2 Mpro, even more so than many of the published proposed inhibitors for SARS-CoV-2 Mpro $[40,44,45]$. Its strong binding affinity could be attributed to its ability to engage with the Mpro via a large number of hydrogen bonds as well as a covalent bond with key cysteine 145 residue. RMH148 also has an excellent safety profile, making it worthy of further optimization. However, RMH148 has two issues that should be addressed: it has low GIT absorption and challenging synthetic feasibility. Both of these issues could be successfully handled by simplifying the structure while maintaining the key features required for interactions. Thus, in the near future, our team will apply deferent drug design techniques that identify the most suitable functional groups, such as 3D pharmacophore, to optimize the generated hit and to produce an easily synthesized analogue worthy of entering clinical trials for management of COVID-19.

\section{Conclusions}

In the current work, a protocol of structure-based drug design was employed with the prime aim of designing a potential specific inhibitor for the SARS-CoV-2 main protease (Mpro) enzyme. The published crystal structure of Mpro (PDB ID: 6y2g) was used in the calculations. Firstly, after native ligand omission, a fragment-based drug design method was employed, using Discovery Studio software and its accompanied default fragment database. The highest ranked five fragments were linked to the alpha-ketoamide retrieved from the co-crystalized ligand, using suitable carbon linkers to yield compound RMH148. The strength of the interaction and mode of binding between RMH148 and the Mpro enzyme were predicted by the covalent docking strategy, using Docktite wizard of MOE 2015 software. Compound RMH148 achieved a higher docking score compared to the co-crystalized ligand, indicating potentially strong binding between RMH148 and the Mpro enzyme. The last simulation step involved three MD simulation experiments with a production phase of $150 \mathrm{~ns}$, which revealed higher stability for the Mpro-RMH148 complex than the free enzyme and Mpro-O6K complex, validating our initial results from the docking step. The cumulative data obtained from this work introduce RMH148 as a possible inhibitor for Mpro. These modeling outcomes may provide the grounds for a lead optimization program for the introduction of RMH148-based COVID-19 inhibitors.

Supplementary Materials: The following are available online at https:/ /www.mdpi.com/article/10 $.3390 /$ pr9061004/s1.

Author Contributions: In this work all authors contributed significantly to the manuscript. Conceptualization, M.A.E.H. and W.M.E.; methodology, M.A.E.H. and W.M.E.; software, M.A.E.H. and W.M.E.; validation, A.A., R.O.E. and H.M.A.; formal analysis, M.F. and M.K.A.-H.A.; investigation, M.A.E.H., S.T.A.-R. and W.M.E.; resources, S.T.A.-R. and W.M.E.; data curation, A.A., R.O.E. and H.M.A.; writing—original draft preparation, M.A.E.H. and M.F.; writing—review and editing, and M.K.A.-H.A. and W.M.E.; visualization; supervision, W.M.E.; project administration, S.T.A.-R. and W.M.E.; funding acquisition, A.A., R.O.E., S.T.A.-R. and H.M.A. All authors have read and agreed to the published version of the manuscript.

Funding: The authors would like to extend their sincere appreciation to the Deanship of Scientific Research at King Saud University for the funding of this research through the Research Group Project No. RG-1439-065.

Institutional Review Board Statement: Not applicable.

Informed Consent Statement: Not applicable.

Data Availability Statement: All the data presented in this study are available in the article body and the supplementary material.

Conflicts of Interest: The authors declare no conflict of interest. 


\section{References}

1. Wang, M.; Cao, R.; Zhang, L.; Yang, X.; Liu, J.; Xu, M.; Shi, Z.; Hu, Z.; Zhong, W.; Xiao, G. Remdesivir and chloroquine effectively inhibit the recently emerged novel coronavirus (2019-nCoV) in vitro. Cell Res. 2020, 30, 269-271. [CrossRef]

2. Zumla, A.; Chan, J.F.W.; Azhar, E.I.; Hui, D.S.C.; Yuen, K.-Y. Coronaviruses-Drug discovery and therapeutic options. Nat. Rev. Drug Discov. 2016, 15, 327-347. [CrossRef]

3. De Wit, E.; Van Doremalen, N.; Falzarano, D.; Munster, V.J. SARS and MERS: Recent insights into emerging coronaviruses. Nat. Rev. Microbiol. 2016, 14, 523-534. [CrossRef]

4. Song, Z.; Xu, Y.; Bao, L.; Zhang, L.; Yu, P.; Qu, Y.; Zhu, H.; Zhao, W.; Han, Y.; Qin, C. From SARS to MERS, Thrusting Coronaviruses into the Spotlight. Viruses 2019, 11, 59. [CrossRef]

5. Borgio, J.F.; Alsuwat, H.S.; Al Otaibi, W.M.; Ibrahim, A.M.; Almandil, N.B.; Al Asoom, L.I.; Salahuddin, M.; Kamaraj, B.; AbdulAzeez, S. State-of-the-art tools unveil potent drug targets amongst clinically approved drugs to inhibit helicase in SARSCoV-2. Arch. Med Sci. 2020, 16, 508-518. [CrossRef]

6. Li, G.; De Clercq, E. Therapeutic options for the 2019 novel coronavirus (2019-nCoV). Nat. Rev. Drug Discov. 2020, 19, 149-150. [CrossRef]

7. Jeong, G.U.; Song, H.; Yoon, G.Y.; Kim, D.; Kwon, Y.-C. Therapeutic Strategies Against COVID-19 and Structural Characterization of SARS-CoV-2: A Review. Front. Microbiol. 2020, 11, 1723. [CrossRef] [PubMed]

8. Cameron, C.E.; Castro, C. The mechanism of action of ribavirin: Lethal mutagenesis of RNA virus genomes mediated by the viral RNA-dependent RNA polymerase. Curr. Opin. Infect. Dis. 2001, 14, 757-764. [CrossRef]

9. Han, D.P.; Penn-Nicholson, A.; Cho, M.W. Identification of critical determinants on ACE2 for SARS-CoV entry and development of a potent entry inhibitor. Virology 2006, 350, 15-25. [CrossRef]

10. Hilgenfeld, R. From SARS to MERS: Crystallographic studies on coronaviral proteases enable antiviral drug design. FEBS J. 2014, 281, 4085-4096. [CrossRef] [PubMed]

11. Ziebuhr, J.; Gorbalenya, A.; Snijder, E. Virus-encoded proteinases and proteolytic processing in the Nidovirales. J. Gen. Virol. 2000, 81, 853-879. [CrossRef] [PubMed]

12. Hegyi, A.; Ziebuhr, J. Conservation of substrate specificities among coronavirus main proteases. J. Gen. Virol. 2002, 83, 595-599. [CrossRef]

13. Du, Q.-S.; Wang, S.-Q.; Zhu, Y.; Wei, D.-Q.; Guo, H.; Sirois, S.; Chou, K.-C. Polyprotein cleavage mechanism of SARS CoV Mpro and chemical modification of the octapeptide. Peptides 2004, 25, 1857-1864. [CrossRef]

14. Pillaiyar, T.; Manickam, M.; Namasivayam, V.; Hayashi, Y.; Jung, S.-H. An Overview of Severe Acute Respiratory SyndromeCoronavirus (SARS-CoV) 3CL Protease Inhibitors: Peptidomimetics and Small Molecule Chemotherapy. J. Med. Chem. 2016, 59, 6595-6628. [CrossRef]

15. Huang, C.; Wei, P.; Fan, K.; Liu, Y.; Lai, L. 3C-like Proteinase from SARS Coronavirus Catalyzes Substrate Hydrolysis by a General Base Mechanism. Biochemistry 2004, 43, 4568-4574. [CrossRef] [PubMed]

16. De Leuw, P.; Stephan, C. Protease inhibitors for the treatment of hepatitis C virus infection. GMS Infect. Dis. 2017, 5, 8.

17. Wang, Y.; Lv, Z.; Chu, Y. HIV protease inhibitors: A review of molecular selectivity and toxicity. HIV/AIDS Res. Palliat. Care 2015, 7, 95-104. [CrossRef]

18. Agbowuro, A.A.; Huston, W.M.; Gamble, A.B.; Tyndall, J.D.A. Proteases and protease inhibitors in infectious diseases. Med. Res. Rev. 2018, 38, 1295-1331. [CrossRef]

19. De Kloe, G.E.; Bailey, D.; Leurs, R.; de Esch, I.J. Transforming fragments into candidates: Small becomes big in medicinal chemistry. Drug Discov. Today 2009, 14, 630-646. [CrossRef]

20. Blum, L.C.; Reymond, J.-L. 970 Million Druglike Small Molecules for Virtual Screening in the Chemical Universe Database GDB-13. J. Am. Chem. Soc. 2009, 131, 8732-8733. [CrossRef]

21. Roughley, S.D.; Hubbard, R.E. How Well Can Fragments Explore Accessed Chemical Space? A Case Study from Heat Shock Protein 90: Miniperspective. J. Med. Chem. 2011, 54, 3989-4005. [CrossRef]

22. Mortenson, P.N.; Murray, C.W. Assessing the lipophilicity of fragments and early hits. J. Comput. Mol. Des. 2011, $25,663-667$. [CrossRef]

23. Kumar, A.; Voet, A.; Zhang, K. Fragment Based Drug Design: From Experimental to Computational Approaches. Curr. Med. Chem. 2012, 19, 5128-5147. [CrossRef]

24. El Hassab, M.A.; Shoun, A.A.; Al-Rashood, S.T.; Al-Warhi, T.; Eldehna, W.M. Identification of a New Potential SARS-COV-2 RNA-Dependent RNA Polymerase Inhibitor via Combining Fragment-Based Drug Design, Docking, Molecular Dynamics, and MM-PBSA Calculations. Front. Chem. 2020, 8, 584894. [CrossRef] [PubMed]

25. Kirsch, P.; Hartman, A.M.; Hirsch, A.K.H.; Empting, M. Concepts and Core Principles of Fragment-Based Drug Design. Molecules 2019, 24, 4309. [CrossRef]

26. Evensen, E.; Joseph-McCarthy, D.; Karplus, M. MCSS Version 2.1; Harvard University: Cambridge, MA, USA, 1997.

27. Rimmert, B.; Sabet, S.; Ackad, E.; Yousef, M.S. A 3D structural model and dynamics of hepatitis C virus NS3/4A protease (genotype 4a, strain ED43) suggest conformational instability of the catalytic triad: Implications in catalysis and drug resistivity. J. Biomol. Struct. Dyn. 2013, 32, 950-958. [CrossRef] [PubMed] 
28. El-Hasab, M.A.E.-M.; El-Bastawissy, E.E.; El-Moselhy, T.F. Identification of potential inhibitors for HCV NS3 genotype 4a by combining protein-ligand interaction fingerprint, 3D pharmacophore, docking, and dynamic simulation. J. Biomol. Struct. Dyn. 2018, 36, 1713-1727. [CrossRef] [PubMed]

29. El-Hassab, M.A.E.-M.; El-Bastawissy, E.E.; El-Moselhy, T.F. Identification of potential inhibitors for HCV NS5b of genotype 4a by combining dynamic simulation, protein-ligand interaction fingerprint, 3D pharmacophore, docking and 3D QSAR. J. Biomol. Struct. Dyn. 2019, 38, 4521-4535. [CrossRef]

30. Nagarajan, H.; Narayanaswamy, S.; Vetrivel, U. Mutational landscape screening of methylene tetrahydrofolate reductase to predict homocystinuria associated variants: An integrative computational approach. Mutat. Res. Mol. Mech. Mutagen. 2020, 819-820, 111687. [CrossRef]

31. Kumari, R.; Kumar, R.C. Open Source Drug Discovery. J. Chem. Inf. Model. 2014, 54, 1951-1962. [CrossRef]

32. Dassault Systèmes BIOVIA. BIOVIA Workbook, Release 2016, BIOVIA Pipeline Pilot, Release 2016; Dassault Systèmes: San Diego, CA, USA, 2016.

33. Böhm, H.-J. The computer program LUDI: A new method for the de novo design of enzyme inhibitors. J. Comput. Mol. Des. 1992, 6, 61-78. [CrossRef]

34. Caflisch, A.; Miranker, A.; Karplus, M. Multiple copy simultaneous search and construction of ligands in binding sites: Application to inhibitors of HIV-1 aspartic proteinase. J. Med. Chem. 1993, 36, 2142-2167. [CrossRef]

35. Cozza, G.; Moro, S. Medicinal Chemistry and the Molecular Operating Environment (MOE): Application of QSAR and Molecular Docking to Drug Discovery. Curr. Top. Med. Chem. 2008, 8, 1555-1572. [CrossRef]

36. Scholz, C.; Knorr, S.; Hamacher, K.; Schmidt, B. DOCKTITE A Highly Versatile Step-by-Step Workflow for Covalent Docking and Virtual Screening in the Molecular Operating Environment. J. Chem. Inf. Model. 2015, 23, 398-406. [CrossRef]

37. Kumalo, H.M.; Bhakat, S.; Soliman, M.E.S. Theory and Applications of Covalent Docking in Drug Discovery: Merits and Pitfalls. Molecules 2015, 20, 1984-2000. [CrossRef]

38. Abraham, M.J.; Murtola, T.; Schulz, R.; Páll, S.; Smith, J.C.; Hess, B.; Lindahl, E. GROMACS: High performance molecular simulations through multi-level parallelism from laptops to supercomputers. SoftwareX 2015, 1-2, 19-25. [CrossRef]

39. Malde, A.K.; Zuo, L.; Breeze, M.; Stroet, M.; Poger, D.; Nair, P.; Oostenbrink, C.; Mark, A.E. An Automated Force Field Topology Builder (ATB) and Repository: Version 1.0. J. Chem. Theory Comput. 2011, 7, 4026-4037. [CrossRef]

40. Bhardwaj, V.K.; Singh, R.; Sharma, J.; Rajendran, V.; Purohit, R.; Kumar, S. Identification of bioactive molecules from Tea plant as SARS-CoV-2 main protease inhibitors. J. Biomol. Struct. Dyn. 2020, 1-13. [CrossRef] [PubMed]

41. Chiu, S.-W.; Pandit, S.A.; Scott, H.L.; Jakobsson, E. An Improved United Atom Force Field for Simulation of Mixed Lipid Bilayers. J. Phys. Chem. B 2009, 113, 2748-2763. [CrossRef] [PubMed]

42. Berendsen, H.J.C.; Postma, J.P.M.; Van Gunsteren, W.F.; DiNola, A.; Haak, J.R. Molecular dynamics with coupling to an external bath. J. Chem. Phys. 1984, 81, 3684-3690. [CrossRef]

43. Parrinello, M.; Rahman, A. Polymorphic transitions in single crystals: A new molecular dynamics method. J. Appl. Phys. 1981, 52, 7182-7190. [CrossRef]

44. Ghahremanpour, M.M.; Tirado-Rives, J.; Deshmukh, M.; Ippolito, J.A.; Zhang, C.H.; Cabeza de Vaca, I.; Liosi, M.E.; Anderson, K.S.; Jorgensen, W.L. Identification of 14 known drugs as inhibitors of the main protease of SARS-CoV-2. ACS Med. Chem. Lett. 2020, 11, 2526-2533. [CrossRef] [PubMed]

45. Havranek, B.; Islam, S.M. Anin silicoapproach for identification of novel inhibitors as potential therapeutics targeting COVID-19 main protease. J. Biomol. Struct. Dyn. 2020, 1-12. [CrossRef] 